\title{
Fisheries stock assessment and decision analysis: the Bayesian approach
}

\author{
ANDRÉ E. PUNT ${ }^{1 *}$ and RAY HILBORN ${ }^{2}$ \\ ${ }^{1}$ Division of Marine Research, CSIRO, GPO Box 1538, Hobart, Tas 7000, Australia. E-mail: \\ Andre.Punt@ml.csiro.au \\ ${ }^{2}$ School of Fisheries, Box 357980, University of Washington, Seattle, WA 98195-7980, USA
}

\section{Contents}

$\begin{array}{ll}\text { Abstract } & \text { page } 35\end{array}$

Introduction $\quad 36$

Evaluating the consequences of management actions $\quad 37$

Choice of alternative hypotheses and their probabilities

Specifying the management options

Calculating performance indices

Presenting the results

Methods for assigning weights to alternative hypotheses

Specifying prior distributions

Noninformative or informative priors

Eliciting expert opinion and using data from other stocks

The prior for $B_{0}$

Expressing the data in the form of a likelihood

Abundance indices

Age-structure data

Current applications

Discussion

Advantages and disadvantages of the Bayesian approach

Future needs and issues

Acknowledgements

Appendix A: Methods for numerical integration

Grid search

The Metropolis algorithm (Hastings, 1970)

The SIR algorithm (Rubin, 1987; Van Dijk et al., 1987)

Appendix B: The age-structured model

References

\begin{abstract}
The Bayesian approach to stock assessment determines the probabilities of alternative hypotheses using information for the stock in question and from inferences for other

*Author to whom correspondence should be addressed.

0960-3166 (C) 1997 Chapman \& Hall
\end{abstract}


stocks/species. These probabilities are essential if the consequences of alternative management actions are to be evaluated through a decision analysis. Using the Bayesian approach to stock assessment and decision analysis it becomes possible to admit the full range of uncertainty and use the collective historical experience of fisheries science when estimating the consequences of proposed management actions. Recent advances in computing algorithms and power have allowed methods based on the Bayesian approach to be used even for fairly complex stock assessment models and to be within the reach of most stock assessment scientists. However, to avoid coming to ill-founded conclusions, care must be taken when selecting prior distributions. In particular, selection of priors designed to be noninformative with respect to quantities of interest to management is problematic. The arguments of the paper are illustrated using New Zealand's western stock of hoki, Macruronus novaezelandiae (Merlucciidae) and the Bering-ChukchiBeaufort Seas stock of bowhead whales as examples.

\section{Introduction}

The most important reason to conduct fisheries stock assessments is to be able to evaluate the consequences of alternative management actions (i.e. to conduct a decision analysis). There are usually competing hypotheses about the dynamics and state of a fishery, and the consequences of management actions may differ depending on which hypothesis is true. The purpose of this paper is to review the Bayesian approach to fisheries stock assessment which we believe offers conceptual simplicity and elegance and can formally and rigorously incorporate expert judgment and inferences from data for other species or stocks. This approach can therefore address the issue of assigning probabilities to alternative hypotheses in a general and complete manner.

Bayesian stock assessment methods use information from two sources to assign probabilities to alternative hypotheses. The first source is data from observations of the stock in question (trends in catch rate, age-composition data, etc.) and the second source is information based on inferences for other stocks/species. The first of these sources is represented in the form of a likelihood function and the information from the second source through prior probability distributions. Bayes Theorem (see Equations 1 and 2 below) is used to combine the information from these sources to obtain the posterior probability for each alternative hypothesis.

The paper is divided into four major sections. In the first, we present an overview of the generic process of evaluating the consequences of alternative management actions and outline several approaches to stock assessment which can be used to provide the information needed to conduct such an evaluation (including the Bayesian approach). In the second, we show how data from other stocks can be used to formulate prior distributions for model parameters within a Bayesian assessment and discuss the merits of trying to formulate noninformative prior distributions which attempt to let the data 'speak for themselves' and dominate the process of assigning probabilities. We highlight the problems with the choice of the prior distribution which is used to summarize the information about the fishery before incorporation of any assessment data. We also review the major computational problems associated with Bayesian fisheries assessment techniques (i.e. the need to integrate across many parameters), and Appendix A describes three algorithms we have found useful.

In the third section, assessments of New Zealand's western stock of hoki 
(Macruronus novaezelandiae, Merlucciidae) and the Bering-Chukchi-Beaufort Seas stock of bowhead whales (Balaena mysticetus, Balaenidae) are used to illustrate some of the points raised in the paper. These assessments should be considered to be examples only - the reports of the New Zealand Stock Assessment plenaries and the International Whaling Commission should be consulted for more up-to-date results. The assessments are based on fully age-structured population dynamics models. Although the use of age-structured models (e.g. Appendix B) complicates the presentation, most of the Bayesian stock assessments that are used for management purposes are based on such models.

It is important to recognize that the Bayesian approach is independent of the population dynamics model and the likelihood used to describe the observations, and is best thought of as a method for describing uncertainty and using historical experience. Thus, with the Bayesian approach, the analyst is free to deal with arbitrarily complex models as long as it is possible to provide prior distributions for the parameters. The techniques described in this paper can easily be applied to simpler models than that in Appendix B, such as surplus production models (e.g. Schaefer 1954, 1957), delaydifference models (Deriso, 1980; Schnute, 1985), or even VPA-based methods such as ADAPT (Gavaris, 1988; Powers and Restrepo, 1992). Hoenig et al. (1994) present Bayesian assessments based on an equilibrium production model while Walters and Punt (1994) consider ADAPT within a Bayesian framework.

The final section contains a discussion of the key advantages and disadvantages of the Bayesian approach as well as the future data collection and analysis requirements for making Bayesian assessments more useful. At various points in the paper, we make suggestions based on our experience applying the techniques discussed.

\section{Evaluating the consequences of management actions}

A decision analysis involves the following five steps:

1. identifying alternative hypotheses about the population dynamics (often referred to as 'states of nature'), $H_{i}$;

2. determining the relative weight of evidence in support of each alternative hypothesis expressed as a relative probability, $P\left(H_{i}\right)$;

3. identifying each alternative management action, $A_{j}$;

4. evaluating the distribution and expected value of each performance measure, $I_{k}$, given the management actions and the hypotheses; and

5. presenting the results to the decision makers.

When there are discrete alternative hypotheses and management actions, a 'decision table' (e.g. Table 1) is an effective aid to summarizing the process of evaluating management actions, and to presenting the results to the decision makers. The alternative hypotheses, $H_{i}$, are given in the first row, the probability assigned to each hypothesis, $P\left(H_{i}\right)$, is given in the second row, the alternative management actions, $A_{j}$, are shown in the leftmost column, and the consequences (in terms of some performance measure $I_{k}$ ), of alternative action $A_{j}$ if a hypothesis $H_{i}$ is true (denoted by $I_{k}\left(H_{i}, A_{j}\right)$ ), are shown in the $(j, i)$ cells. The rightmost column lists the expected value of $I_{k}$ for each management 
Table 1. A simple decision table to evaluate the consequences, in terms of the ratio of the stock size at the end of the management period to the virgin biomass, of a variety of alternative future annual catch quotas (from Hilborn et al., 1994)

\begin{tabular}{lllllllll}
\hline & \multicolumn{9}{c}{ Alternative hypotheses } & (virgin biomass 3 & $\left.10^{3} \mathrm{t}\right)^{1}$ & & Expectation \\
\cline { 2 - 6 } \cline { 5 - 6 } Quota $\left(10^{3} \mathrm{t}\right)$ & 750 & 950 & 1150 & 1350 & 1550 & 1750 & \multirow{2}{*}{$1047^{2}$} \\
\hline 100 & $(0.099)$ & $(0.465)$ & $(0.317)$ & $(0.096)$ & $(0.020)$ & $(0.003)$ & \\
150 & $0.51^{3}$ & 0.63 & 0.70 & 0.75 & 0.78 & 0.81 & $0.66^{4}$ \\
200 & 0.26 & 0.45 & 0.56 & 0.63 & 0.69 & 0.72 & 0.49 \\
\hline
\end{tabular}

${ }^{1}$ Values in parentheses are the probabilities assigned to each hypothesis.

${ }^{2}$ Expressed as biomass $\left(10^{3} \mathrm{t}\right)$.

${ }^{3}$ This entry implies that if the virgin biomass is $750 \times 10^{3} \mathrm{t}$ and a quota of $100 \times 10^{3} \mathrm{t}$ is set for 5 years, the biomass at the end of the 5 years will be $51 \%$ of the virgin biomass.

${ }^{4}$ The expectation for a quota is calculated by multiplying the ratios for that quota by the probabilities associated with the corresponding virgin biomasses, i.e. $0.66=0.099 \times 0.51+0.465 \times 0.63+\ldots$.

action (i.e. $\left.\sum_{i} I_{k}\left(A_{j}, H_{i}\right) P\left(H_{i}\right)\right)$. There are often several measures of performance and separate decision tables must be produced for each.

Each one of steps (1-5) involves a number of philosophical or methodological decisions.

\section{CHOICE OF ALTERNATIVE HYPOTHESES AND THEIR PROBABILITIES}

The choice of alternative hypotheses (1) is usually a question of preference and judgment. The 'alternative hypotheses' consist of all possible structural models combined with all values for the parameters of those models. The most common approach is to select a single structural model and to consider the uncertainty in its parameters only. A more defensible alternative is to consider a series of truly different structural models. However, apart from being computationally more intensive, it is difficult to 'bound' the range of models considered. A related issue is how to determine how many model parameters should be considered uncertain.

These choices are closely related to step (2), the assignment of probabilities to alternative hypotheses which is dealt with in detail below. It is not possible to consider (or even list) all possible hypotheses, because there is an infinite number. Therefore, any decision analysis must exclude most plausible hypotheses by asserting that they have little or no credibility relative to the models that are considered (i.e. $P\left(H_{i}\right) \cong 0$ ). Some of the hypotheses excluded clearly have a non-zero probability. For instance, systematic environmental change is now an acknowledged component of aquatic ecosystems (Cushing, 1982; Hilborn and Walters, 1992), yet very few decision analyses allow for this.

\section{SPECIFYING THE MANAGEMENT OPTIONS}

Management actions are generally arrived at through discussion between managers, user groups and scientists, and are usually quite simple (alternative series of future catches or exploitation rates, size limits, etc.). But as management systems become more sophisticated, actions may take the form of feedback-control 'decision rules'. A decision rule defines a management action as a function of the estimated current status of the 
stock and perhaps even of the uncertainty about its estimated status (Hilborn and Luedke, 1987; Sainsbury, 1988; IWC, 1989; Butterworth and Bergh, 1993). The International Whaling Commission (IWC) has conducted the most thorough examination of the performance of alternative decision rules to date (e.g. IWC, 1989). Other analyses to contrast alternative decision rules for specific stocks are those for South African anchovy (Engraulis capensis, Clupeidae) by Bergh and Butterworth (1987), Butterworth and Bergh (1993) and Butterworth et al. (1993), yellowtail flounder (Limanda ferruginea, pleuronectidae) off eastern Canada by Collie and Walters (1991), the Cape hakes (Merluccius capensis and M. paradoxus, Merlucciidae) by Punt (1993) and Punt et al. (1995b), North Sea cod (Gadus morhua, Gadidae) by Pelletier and Laurec (1992), and Australian orange roughy (Hoplostethus atlanticus, Trachichthyidae) by Smith (1993).

The population dynamics model used when comparing decision rules is often referred to as an 'operating model' (e.g. Punt, 1992) and can be very complex in order to mimic the dynamics of the system for which a decision rule is being sought. For example, to compare decision rules for Southern Hemisphere minke whales, IWC (1993) developed a model which accounts for multiple stocks as well as multiple whaling grounds.

\section{CALCULATING PERFORMANCE INDICES}

The consequences of alternative management actions, given a specific hypothesis, can be determined analytically for very simple models, but for most fisheries problems they must be computed by Monte Carlo simulation. The objective is to calculate the future stock size $(N)$, given the time series of future catches $\left(C_{y}\right)$, dictated by management actions and any environmental fluctuations $(\mathrm{y})$. In the simplest case, where the catches are pre-specified and do not depend on future stock size, the calculation involves generating y from its assumed distribution (usually log-normal) and using equations such as those in Appendix B to project the population forward. The resultant values for $N$ and $C_{y}$ provide the basis for the calculation of the performance measures (average catch, changes in catch, variance of catch, average stock size, changes in stock size, the average and variance of exploitation rate, etc.).

Projection is more difficult when the management action for a given year is determined by a decision rule and hence depends in some way on the system state in that year. For instance, many management policies now used for large-scale industrial fisheries are based on attempting to fix the exploitation rate (for example to $F_{0.1}$ ). The quota for a given year is obtained by multiplying the estimate of exploitable stock size during that year by the desired exploitation rate. Modelling the assessment procedure is necessary to evaluate this sort of decision rule (e.g. Punt, 1993; McAllister, 1995). This is a highly laborious process that, in the end, usually results in the need for considerable simplification or short cuts. One such short cut is to assume that the estimated stock size $\left(\hat{B}_{y}\right)$ is distributed about the true stock size $\left(B_{y}\right)$ with some error such as $\hat{B}_{y}=B_{y} \exp \left(v_{y}\right)$, and then setting the annual quota based on a target exploitation rate $u$ : $C_{y}=u \hat{B}_{y}$. This requires specifying a sequence of random errors in estimating stock size (the $v_{y}$ ).

\section{PRESENTING THE RESULTS}

Fisheries models can produce many performance measures, such as average catch, variance of catch, average stock size, minimum stock size, or probability of falling below some threshold level. In any specific case, these measures are chosen to quantify the 
management objectives. Much of the art of stock assessment involves determining what to present to managers and the best ways to summarize the information. The papers in Smith et al. (1993) illustrate the diversity in what different scientists present to managers. When dealing with discrete hypotheses or a small number of values for a single parameter, the decision table format of Table 1 is an effective means of presentation, though multiple tables are required if there is more than one performance measure. If the decision makers formulate a true objective function (Hilborn and Walters, 1992), the results of the decision table can be compressed to the expected utility for each possible management action. This rarely happens and we believe that most fisheries management groups should discuss the trade-offs between alternative performance measures explicitly. Participants in the decision process often have competing objectives and the most scientists can hope for is to present the distribution of consequences of management alternatives and let the decision process lead to decisions. In many cases stock assessment scientists have some role as decision advisors, and should, at the very least, make sure that the decision makers understand the consequences of alternative management actions.

When there is uncertainty in several parameters, the decision table format is too limiting because it is impossible to express all the hypotheses about several parameters as alternative states of nature. The approach we have used is to aggregate outcomes across alternative hypotheses and simply present the marginal distribution of consequences for each alternative action. For example, Fig. 1 shows the distribution of the biomass of hoki after 5 years of a catch of $300000 \mathrm{t}$ beginning in 1995 . We have found that managers gravitate toward the simplest presentation and that the expected value of consequences (the arrow in Fig. 1) is often all they want to see. Although the expected values summarize the central tendency of the distributions, some scenarios

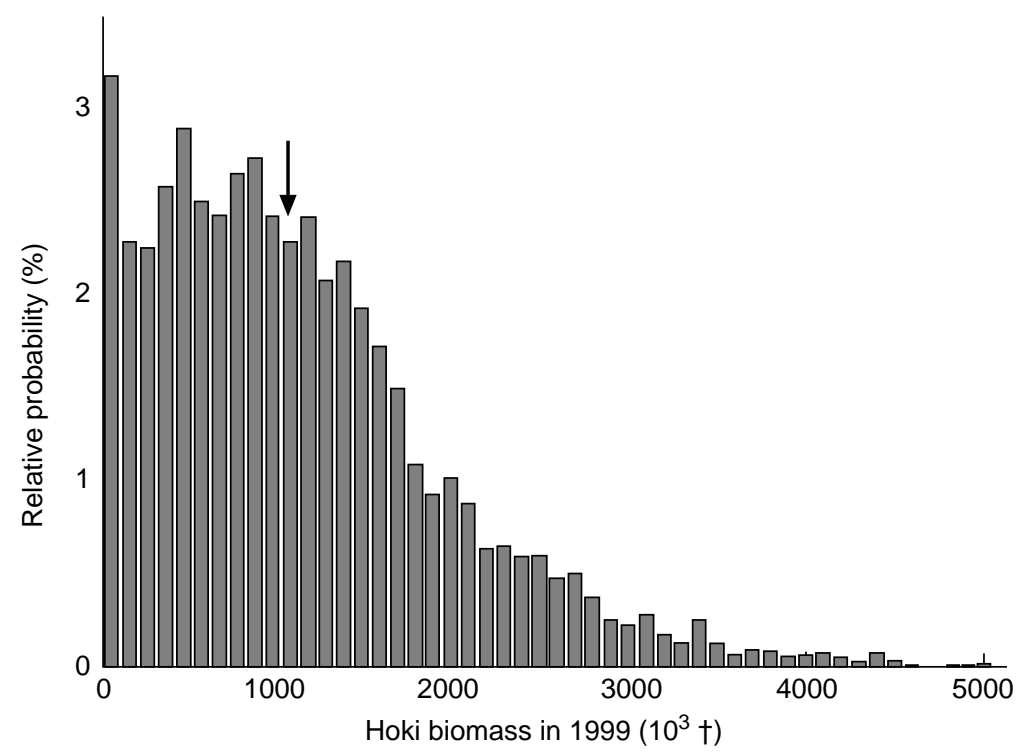

Fig. 1. Distribution of the biomass of hoki after 5 years of a catch of $300000 \mathrm{t}$ beginning in 1995 . The arrow indicates the expected value. 
that have low probability may have disastrous consequences and this should be shown clearly to decision makers.

The International Whaling Commission adopted a different way of interpreting the results of projections, namely specifying minimum levels of performance for some key variables (e.g. the time to recover to a pre-specified threshold level). Any decision rules that did not satisfy these criteria for any state of nature were automatically rejected, no matter how well they performed on other measures or for other states of nature. This is effectively the same as having an objective that says any policy with these management outcomes is unacceptable.

\section{Methods for assigning weights to alternative hypotheses}

The most difficult element in a decision analysis is assigning weights to alternative hypotheses (different models and values for parameters within models). The choice of models must be considered explicitly in this process and each model must be assigned a weight because if a model is not considered, it is implicitly given zero weight. In this section we first review alternative methods that have been used to assign probabilities and then explore in detail how this is done in a Bayesian assessment.

There are several competing methods to assign relative weights to alternative hypotheses. The traditional and simplest is to select a single model, set its parameters to the values which fit the available data 'best', and ignore all other models/parameter values (i.e. $P\left(H_{i}\right)=1$ for the selected model/'best fit' parameter values and $P\left(H_{i}\right)=0$ for all other models/parameter values). An extension of this approach is to consider several models and assign them equal probability (Punt and Butterworth, 1991). The values for the model parameters are determined as before but no account is taken of parameter uncertainty. Although this is better than the traditional approach, the possibility that the models do not have the same credibility is ignored.

Bootstrap or Monte Carlo methods (Francis, 1992; Restrepo et al., 1992) use resampling to calculate frequency distributions for the values of the parameters of a pre-specified model. These distributions are then used as if they represented the probabilities of alternative hypotheses. To date, these methods have been used only in conjunction with a single model and have not been used to compare structurally different models.

Bayesian analysts compute the relative probability of alternative hypotheses using the information contained in the prior probability distribution for each state of nature, and the goodness of fit to the available data. These two sources of information are combined using Bayes Theorem. If the alternative hypotheses can be described by a vector of continuous parameters $(\underline{\underline{t}})$, the posterior probability is calculated using the continuous form of Bayes Theorem:

$$
(\underline{\mathfrak{t}}) d \underline{\mathrm{t}}=\frac{L(D \mid \underline{\mathrm{t}}) p(\underline{\mathrm{t}}) d \underline{\mathrm{t}}}{\int L(D \mid \underline{\lfloor} 9) p(\underline{\mathrm{t} 9}) d \underline{\mathrm{t}} 9}
$$

where $(\underline{\mathfrak{t}})$ is the posterior probability of the vector $\underline{\mathfrak{g}}$ given the data, $L(D \mid \underline{\mathfrak{t}})$ is the probability (likelihood) of the data given the vector $\underline{\underline{t}}$, and $p(\underline{\underline{\mathfrak{t}}})$ is the prior probability of the vector $\underline{t}$. 
If, however, the alternative hypotheses are considered as a set of discrete possibilities $\left(H_{i}\right)$, then Bayes Theorem is written as:

$$
\left(H_{i}\right)=\frac{L\left(D \mid H_{i}\right) p\left(H_{i}\right)}{\sum_{j} L\left(D \mid H_{j}\right) p\left(H_{j}\right)} .
$$

There are three main reasons for using the Bayesian approach to assigning relative weights: (1) it is straightforward to represent the full range of uncertainty (both parameter uncertainty and model-structure uncertainty); (2) information based on 'expert opinion' and inferences about other species can be incorporated explicitly and rigorously into the stock assessment through the prior distributions; and (3) the output of the analysis is exactly the information needed for decision analysis (i.e. the probability of alternative states of nature). Thus, unlike bootstrap approaches, it is not necessary to argue that the posterior distribution can be assumed to represent probabilities because that is exactly what the Bayesian approach provides.

Walters and Hilborn (1976) first proposed that Bayesian analysis could be used to evaluate alternative fisheries policies, but the first major applications in the context of traditional stock assessment models were Bergh and Butterworth (1987), who considered a single age-structured model with uncertainty in several parameters, and Sainsbury (1988), who considered six structurally different models. Since then, Bayesian methods have been applied to a broad range of stock assessment problems (Collie and Walters, 1991; Thompson, 1992; Givens et al., 1993; Kinas, 1993; Hilborn et al., 1994; McAllister et al., 1994; Punt et al., 1994; Walters and Ludwig, 1994; Walters and Punt, 1994).

Whether a discrete or continuous model (Equation 1 or 2) is used, there are two key elements to the Bayesian analysis - the prior distribution $p(\underline{\mathfrak{t}})$, and the likelihood of the data $L(D \mid \underline{t})$. Selecting the methods to determine these elements is the major problem in formulating a Bayesian analysis and this is discussed in the following two subsections.

\section{SPECIFYING PRIOR DISTRIBUTIONS}

The prior distribution for a parameter (or set of parameters) summarizes the information about that parameter (or parameters) from all knowledge except the data used in the likelihood calculations of the stock assessment. In practice this means using basic biological knowledge or historical information from other stocks to determine a priori weights for alternative states of nature. These states of nature include alternative models as well as different values for the parameters of the models. The parameters of the agestructured model described in Appendix B are: virgin biomass, $B_{0}$, the steepness of the stock-recruit relationship, $h$, the rate of natural mortality, $M$, selectivity at age $a, S_{a}$, fecundity at age $a, f_{a}$, weight at age $a, w_{a}$, and the recruitment multipliers for each year $y, \quad y$. In principle, prior distributions should be specified for all these parameters. However, we often treat some of them as known, especially those well determined by data such as weight at age and fecundity at age. Parameters such as $B_{0}$, which are poorly determined by external information, must be assigned priors.

Developing prior distributions is undoubtedly the most controversial aspect of any Bayesian analysis (Lindley, 1983; Walters and Ludwig, 1994). We therefore strongly 
recommend that whenever a Bayesian assessment is conducted, considerable care should be taken to document fully the basis for the various prior distributions. This documentation process must include specifying which models were considered for inclusion in the analysis and why some of these models were not considered further, even though they may be plausible. The guidelines for ranking hypotheses given by Butterworth et al. (1996) could be helpful as part of the model selection process.

Care needs to be taken not to reject a model simply because it is too complex. This is because it may represent a hypothesis the future behaviour of which is not captured by any simple model. For example, environmental change is now accepted as a major component of fisheries management. Freshwater, estuarine and even marine habitats are changing due to effects of humans, and not only have natural climate shifts been demonstrated to have affected fish production in the past (Cushing, 1982) but they should also be expected to affect it in the future. Most stock assessments ignore environmental change and assume that future environmental effects will be either an average of historical environmental conditions or random with no underlying trend. Once it is admitted that the environment may change systematically (in the medium term) for better or for worse, it will be found that the uncertainty about the future is greater than if it is assumed that the environment shows no directional trend. The possibility of environmental change should therefore be considered when developing a range of plausible models for consideration in a decision analysis.

Care should be taken when selecting the functional form for a prior because poor choices can lead to incorrect inferences. We have also noticed a tendency to underestimate uncertainty, and hence to specify unrealistically informative priors - this tendency should be explicitly acknowledged and avoided. In particular, priors that assign zero probability to some values of a parameter should be selected only if this is a reasonable assumption: if some value is assigned zero probability a priori, Bayes Theorem ensures that the posterior also assigns it zero probability. If the likelihood favours values close to where the prior assigns zero probability, the mode of the posterior may be at its extreme, which is not desirable (e.g. Punt and Butterworth, 1996). We suggest that unless there is a very good reason for not doing so, the prior should assign a non-zero (but possibly very small) probability to all plausuble values.

In many situations, it is not at all obvious which prior is the most appropriate and we suggest that the sensitivity of the results to the choice of the prior be examined and, if necessary, the implications for management reported to the decision makers.

\section{Noninformative or informative priors}

There are two types of priors: informative and noninformative (or 'reference'). Box and Tiao (1973) define a noninformative prior as one that provides little information relative to the experiment - in this case the stock assessment data. Informative prior distributions, on the other hand, summarize the evidence about the parameters concerned from many sources and may well have a considerable impact on the results.

Using informative prior distributions allows the incorporation of information available to stock assessment scientists from the literature and in light of their experience with other stocks. However, using informative priors may lead to problems because of the subjective beliefs of stock assessment scientists. This and other problems have led Walters and Ludwig (1994) to recommend that noninformative priors should be the default choice when performing Bayesian stock assessments. We disagree with 
this argument and believe that, wherever possible, historical experience with other stocks should be used in the assessment. However, even if we wanted to use noninformative priors, the best method for choosing such priors is still an issue of considerable debate (Box and Tiao, 1973; Bernardo, 1979; Lindley, 1983). Jeffreys (1961), Box and Tiao (1973) and Bernardo (1979) describe techniques for obtaining noninformative priors. Unfortunately most of these techniques can, on occasion, lead to noninformative priors that are counter-intuitive or demonstrably in error. So far, they have not been used for age-structured stock assessments. Instead, uniform priors on some measurement system (e.g. uniform, or uniform on a logarithmic scale) are generally chosen when noninformative priors are needed. The use of noninformative priors is controversial because they are sensitive to the choice of measurement system. In almost all stock assessment models any prior will be informative with respect to some quantities of interest even if it is noninformative for others. Take the problem of selecting a prior for $B_{0}$. A uniform prior for $B_{0}$ is noninformative with respect to $B_{0}$, yet it will not be noninformative for other management-related quantities such as the ratio of the current biomass to $B_{0}$ (Fig. 2). This had led some authors (e.g. Lindley, 1983) to suggest that the use of noninformative priors should be abandoned.

We advocate a pragmatic approach to the choice between noninformative and informative priors and have, on occasion, incorporated both types into analyses (e.g. McAllister et al., 1994). We would prefer to select a noninformative prior and test sensitivity to alternatives than 'dream up' an informative prior that perhaps markedly biases the results. On the other hand, well-thought-out informative priors can reduce uncertainty considerably. Indeed, using noninformative priors implies that no information from fisheries science is relevant to the parameter in question. Most existing non-Bayesian assessments fix some parameters (such as natural mortality) for

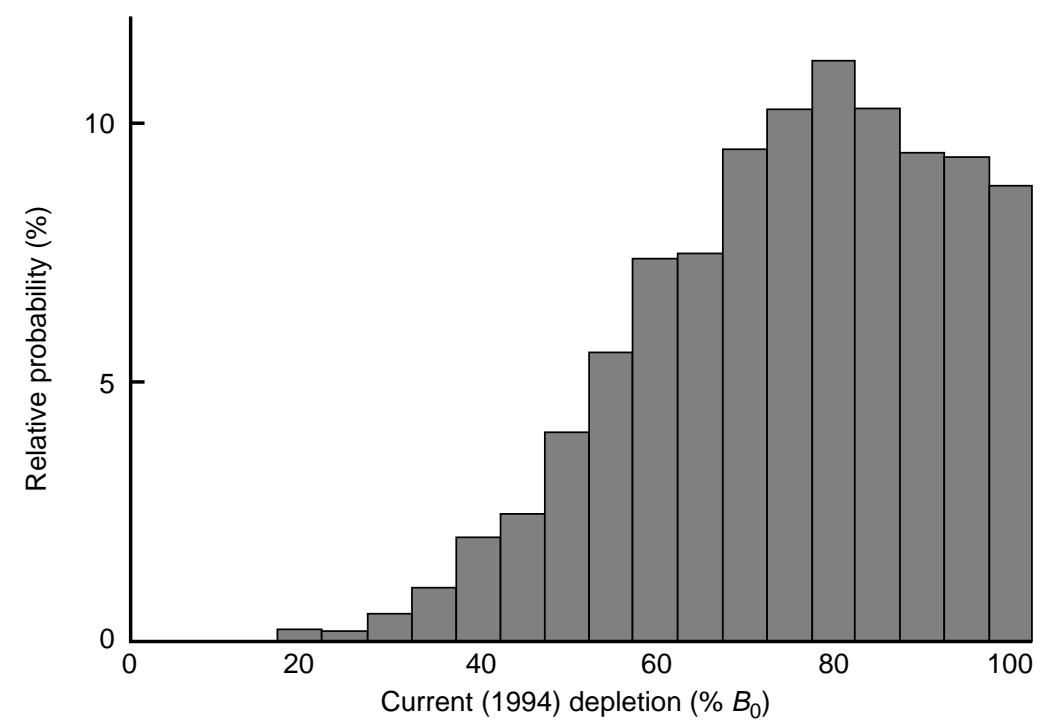

Fig. 2. Posterior distribution for the current (1994) biomass of hoki divided by $B_{0}$ and expressed as a percentage, based on an assessment which ignores the data for the stock concerned and places a $\mathrm{U}[0$, $3000 \mathrm{kt}$ ] prior distribution on $B_{0}$. 
the analysis. To a Bayesian, this implies that there is a very informative prior, i.e. that the parameter is known exactly. Thus, while many non-Bayesians object to the use of priors, they use extremely informative priors in their own analyses.

\section{Eliciting expert opinion and using data from other stocks}

In principle, one of the most powerful methods for developing informative priors is to synthesize the information from a group of experts. IWC (1995) developed priors for the bowhead assessment by consensus. However, Butterworth (1995) pointed out that the a priori correlations among some of the parameters, for example that between $M$ and the age at maturity, are missing. Walters and Ludwig (1994) caution against the use of 'subjective' prior distributions based on arguments from 'basic biology' (fecundity, longevity etc.) rather than on empirical data. Prior distributions for some parameters (such as fecundity at age, weight at age and selectivity at age) can be set equal to their sampling distributions.

We advocate using parameters that do not depend on the parameter which scales the population ( $B_{0}$ in the model described in Appendix B) as often as possible. Such parameters are then comparable among stocks, making it considerably easier to construct priors. For example, it seems reasonable to assume that the prior distributions for $B_{0}$ and steepness (Equation B.5) are uncorrelated (the resilience of the stock-recruit relationship should not depend a priori on the size of the population prior to harvesting). In contrast, it is unreasonable to assume that the parameters $\mathbb{E}$ and (Equation B.5) are independent. Parameters such as steepness can be computed for many stocks using, for example, the data compiled by Myers et al. (1995) and a prior constructed by selecting estimates for stocks that are similar to the one under consideration. Care must be taken to specify how the stocks used were selected and to consider only those stocks for which the assessments are 'reasonable' although what is reasonable is, of course, subjective. For example, Punt et al. (1994) weighted the contribution of each estimate by its variance when constructing a prior for steepness.

The use of data from diverse stocks to formulate a prior is known as meta-analysis. Hierarchical meta-analysis (Gelman et al., 1995) can be used to develop a prior for a parameter from values for that parameter for other stocks under the assumption that the stocks differ in that parameter. For example, it seems reasonable to assume that the steepness for a 'new' groundfish stock will be drawn from a distribution for steepness which could be parameterized using estimates for other groundfish stocks, but that account needs to be taken of uncertainty about the estimates for other stocks and differences in steepness between stocks. Liermann and Hilborn (in press) develop a prior for the parameter which could be used to measure the extent of depensation in the stock-recruitment relationship using meta-analysis. If the raw assessment data for various stocks are available, an empirical Bayes approach could be applied (e.g. Hoenig et al., 1994).

'Selection bias' is a potential problem when developing a prior using data for similar stocks. Assessments in the literature tend to be for large, productive populations (small, less productive populations in general receive less research funding). If the stocks considered are not representative of all similar stocks, an inappropriate prior may be selected. For example, the analyses of Best (1990) which could, in principle, be used to develop a prior distribution for the rate of increase of severely depleted whale populations, were criticized because the stocks included could have been 'self- 
selecting': only stocks that had increased substantially could be monitored because only they were large enough (IWC, 1990). However, a more detailed evaluation of all severely depleted large baleen whale stocks by Best (1993) suggests that this is unlikely to be the case.

\section{The prior for $B_{0}$}

The prior for $B_{0}$ (or more generally the parameter which scales the overall abundance) can have a considerable impact on the final results of any decision analysis. As every population dynamics model, age-structured or otherwise, contains such a parameter, it is of particular importance in most assessments. No one has performed an analysis of the frequency distribution of initial population sizes and the problem of selection bias would be very severe in such an analysis. In principle, a prior for $B_{0}$ could be developed using estimates of $B_{0}$ for the same species in different areas after making allowance for differences in the size of the available habitat areas.

We have found three general approaches to selecting a prior for the parameter which scales the overall abundance. The first is to select a 'base case' prior for $B_{0}$ by consensus and then to conduct an extensive sensitivity examination of this choice. The philosophy behind the other two approaches is to place a prior on the current population size instead of on $B_{0}$ (either the biomass in the current year or the current depletion). A prior for the current abundance could be obtained from a tagging experiment while a $U[0,1]$ prior on current depletion reflects a lack of information about the current status of the resource (it could be anything from unexploited to verging on collapse). Computationally, however, these latter two approaches are much more complex that the first approach. This is because while it is straightforward to project the population dynamics model forward from $B_{0}$ to calculate the biomass trajectory and hence compute the likelihood, it is generally impossible to project the population backwards from a current population size or current depletion. The solution to the problem of calculating the biomass trajectory corresponding to a value for current biomass (or current depletion) is to choose different values for $B_{0}$ and project the model forward until a value is chosen that 'hits' the current biomass value exactly (Butterworth and Punt, 1995).

If the data are sufficiently informative, the choice of the prior for $B_{0}$ will not affect the results substantially. On the other hand, if the results are sensitive to this prior, this sensitivity is indicative of the fact that the data provide little information about one of the most important yield-determining factors, and this constraint must be pointed out to the decision makers.

\section{EXPRESSING THE DATA IN THE FORM OF A LIKELIHOOD}

The priors discussed above are the first element in applying Bayes Theorem; the second element is the probability of the observed data given that each alternative hypothesis is true $L(D \mid \underline{t})$. For most stock assessments, there are two main sources of information. The first is information on trends in population size in the form of surveys or standardized catch-rate data, and the other are data on the age (or size) structure of the catches. The process of specifying the likelihood for each of these data sources will be dealt with in turn. The likelihood for several data sources is the product of the likelihoods for each separate data source if they are independent. 
Abundance indices

Consider the case of an abundance series $\left\{I_{y}\right\}$. This series can be an index either of absolute abundance (such as estimates of spawner biomass from egg surveys or direct counts of population numbers from aerial or visual surveys) or of relative abundance (such as trawl surveys, or catch rates). The deterministic component of the model relating an abundance index to the population dynamics model is of the form $I_{y}=q B_{y}$, where $q$ is the 'catchability coefficient' and $B_{y}$ is the population dynamics quantity that is being indexed (for example, catch rates are usually assumed to be related to exploitable biomass). For series that are assumed to be indices of absolute abundance, $q$ is taken to be equal to 1 . The value of $B_{y}$ is calculated using the population dynamics model and is a function of the parameters of the model and the catch history.

It is necessary to specify the stochastic component of the observation process as well as its deterministic component. The assumption of log-normality guarantees that an observed quantity cannot be negative, something which is certainly true of most indices of abundance, such as the results of trawl surveys and catch rates. This assumption is, therefore, a very common one in stock assessment work.

The likelihood function for abundance index data is of the form:

$$
L=\prod_{y} \frac{1}{\sqrt{2 V_{y}}} \exp \left(-\frac{\left(Y_{y}-U_{y}\right)^{2}}{2 V_{y}}\right)
$$

where, for the situation described above, $Y_{y}=\ln \left(I_{y}\right), U_{y}=\ln \left(q B_{y}\right)$ and $V_{y}$ is the variance of the observation error for data point $Y_{y}$.

For relative abundance series, a prior distribution for the catchability coefficient must be specified. In principle, an informative prior for this parameter can be constructed (e.g. Punt et al., 1994). However, for most relative abundance series, this is not possible and it is necessary to use a prior that is as noninformative as possible. Walters and Ludwig (1994) argue for the use of a uniform prior from 0 to $\infty$ for $q$ and show that this assumption leads to a closed form solution for the integral over the prior (see also Punt and Butterworth, 1996). However, as their Figure 1 indicates, such a prior tends to give larger weight to low values for $B_{0}$ if the data are not informative. While this might be desirable because resultant estimates are conservative (Walters and Ludwig, 1994), this prior is clearly not noninformative. An alternative that favours no values of $B_{0}$ if the data are noninformative is uniform on a logarithmic scale over the 0 to $\infty$ interval and we recommend this approach.

Note that the preceding discussion assumes that a relative abundance index is related linearly to abundance. This need not be the case for many reasons (especially if the index is derived from commercial catch-rate data). It is, however, straightforward to generalize the observation model to examine the implications of non-linearity in the relationship.

\section{Age-structure data}

Age-structure data contain information about the total number of fish landed as well as the age composition of the catch. The total is used implicitly when projecting the population dynamics model forward through Equation B.6 which forces the modelpredicted catch to equal the observed catch. Thus, only the information about the age composition of the catches is included in the likelihood function. It is possible to allow 
for the eventuality that the catches differ from the recorded values by adding an extra component to the likelihood function (Methot, 1989, for details). The observed fraction of the catch (by number) taken in year $y$ consisting of fish of age $a$ is usually assumed to be multinomially distributed about its expected value (although, for an alternative, see Schnute and Richards, 1995), so the contribution of the age-composition data to the loglikelihood function is given by:

$$
L=\prod_{y} \prod_{a} \hat{\rho}_{y, a}^{N_{y}^{e} \rho_{y, a}^{o b s}}
$$

where $\hat{\rho}_{y, a}$ is the model estimate of the fraction of the catch (in number) during year $y$ which consists of fish of age $a, N_{y}^{e}$ is the effective number of fish aged during year $y$, and $\rho_{y, a}^{o b s}$ is the observed fraction of the catch (in number) during year $y$ which consists of fish of age $a$.

Specifying a value for $N_{y}^{e}$ is difficult because the age-composition information does not generally come from a simple random sample from the total catch, but is instead estimated using a length-frequency sample and an age-length key (Ricker, 1975). The usual approach for dealing with the problem of specifying the $N_{y}^{e}$ is to consider a range of values and examine sensitivity to this choice. They could, in principle, however, be set from the results of analyses such as those of Baird (1983) and Gavaris and Gavaris (1983). Equation 4 can be extended straightforwardly to account for uncertainty and bias in the ageing process (Methot, 1989; Richards et al., 1992).

\section{Current applications}

While the basic formulation of Bayes Theorem is both simple and elegant and the theoretical advantages of this approach for fisheries assessments were recognized long ago (Walters and Hilborn, 1976), practical implementation in actual stock assessments requires that priors, models and likelihoods be specified. In this section we overview two stock assessments to explore how Bayesian methods can be applied in practice.

The hoki and bowhead stocks are markedly different in terms of biology and exploitation history. Hoki are fast-growing and short-lived (Sullivan et al., 1995) whereas some bowhead whales probably live to well over 100 years (IWC, 1995). Recruitment of hoki appears to exhibit considerable interannual fluctuations, while the population dynamics of bowhead whales are (probably reasonably) assumed to be deterministic. Catches of hoki exceeded $10000 \mathrm{t}$ for the first time in 1975 and became substantial only in 1986 when $86000 \mathrm{t}$ were landed (Sullivan et al., 1995). In contrast, catches of bowhead whales peaked in 1852 during a period of extensive Yankee whaling (Bockstoce and Botkin, 1983). There is now only a subsistence fishery for bowheads by Native Americans in Alaska. The assessments for both stocks are based on an age- and sex-structured population dynamics model that divides the population into 'available' and 'unavailable' components. Only the hoki assessment allows for fluctuations in recruitment about its expected value (i.e. $y=1$ for the bowhead stock).

The two assessments are based on different data types. The hoki assessment uses catch-rate, trawl survey and acoustic data (Sullivan et al., 1995), whereas the bowhead assessment uses estimates of current population size inferred from visual and acoustic surveys at Point Barrow, Alaska, and estimates of the fraction of the population that 
consisted of calves and mature animals in 1988 and 1989 (IWC, 1995, for details). For both assessments, the observation error variances ( $V_{y}$ in Equation 3$)$ are assumed to be known exactly, so there is no need to specify priors for them.

The hoki assessment places prior distributions on $B_{0}, M$, steepness, the recruitment multipliers, the catchability coefficients and the extent of recruitment variability. The recruitment multipliers are assumed to be lognormally distributed with a median of 1 and a $C V$ of ${ }_{r}$. The prior distribution for the parameter that measures the extent of recruitment fluctuation, $r$, and that for steepness, are derived from analyses of stock and recruitment data for several stocks of gadoids (Punt et al., 1994). The prior for $B_{0}$ was selected by consensus to be $U[0,3000000 \mathrm{t}]$, although the sensitivity of the results to alternative choices for this prior is examined as a routine part of the stock assessment process (Punt et al., 1993, 1994). The prior for $M$ was also selected by consensus and is assumed to be uniform over ranges which depend on sex. The catchability coefficients for the catch rate and trawl survey series are assumed to be uniform on a logarithmic scale due to paucity of data. An informative prior distribution is assumed for the catchability coefficient for the acoustic surveys. This prior distribution was constructed by those involved in the acoustic surveys to quantify the uncertainties associated with acoustic indices as measures of absolute abundance. These uncertainties included uncertainty in the estimate of acoustic target strength, species identification and the proportion of the population in the area surveyed.

For the bowhead assessment, prior distributions are specified for the pre-exploitation size of the population, $K$, the population size at which $M S Y$ is achieved (MSYL), the ratio of $M S Y$ to $M S Y L$ (MSYR), the age at maturity, the survival rate for adults, the survival rate for juveniles, and the age at which animals first experience adult natural mortality (IWC, 1995). These prior distributions were selected after considerable discussion by the Scientific Committee of the IWC. Although Raftery et al. (1995) assert that this was a successful exercise, Butterworth (1995), Butterworth and Punt (1995) and Punt and Butterworth (1996) point out several problems with the priors that were selected, and Baker (1995) raises some concerns about the approach used to derive the data. In particular, two of the priors (those for $K$ and MSYR) are inconsistent because the prodedure used to devise the prior for $K$, which is based on an estimation approach by Delury (1947), effectively assumes that $M S Y R=0$ (Butterworth and Punt, 1995). Wolpert (1995) notes that the use of a deterministic model for the analyses leads to an instance of Borel's Paradox ${ }^{1}$, although Raftery et al. (1995) show by example that, in this case, the extent of error is likely to be small.

The quantity of greatest interest pertaining to the management of the bowhead stock is the current replacement yield (the greatest number of whales that could be taken in a given year without the population decreasing). In the past, the IWC Scientific Committee has based its recommendation for a catch limit on the lower 5th percentile of the sampling distribution for the current replacement yield. In 1994, it based its recommendation on the lower 5th percentile of the posterior probability distribution for the 1994 replacement yield. The lower 5th percentile was chosen because it is conservative in the face of uncertainty. Other quantities that were reported by the Scientific Committee are the current depletion of the female component of the

\footnotetext{
${ }^{1}$ This paradox occurs because the dimensionality of the prior distribution exceeds that of the set of parameters needed to specify the posterior distribution - in such a case, the posterior is non-unique and depends on the scale on which the parameters are expressed.
} 

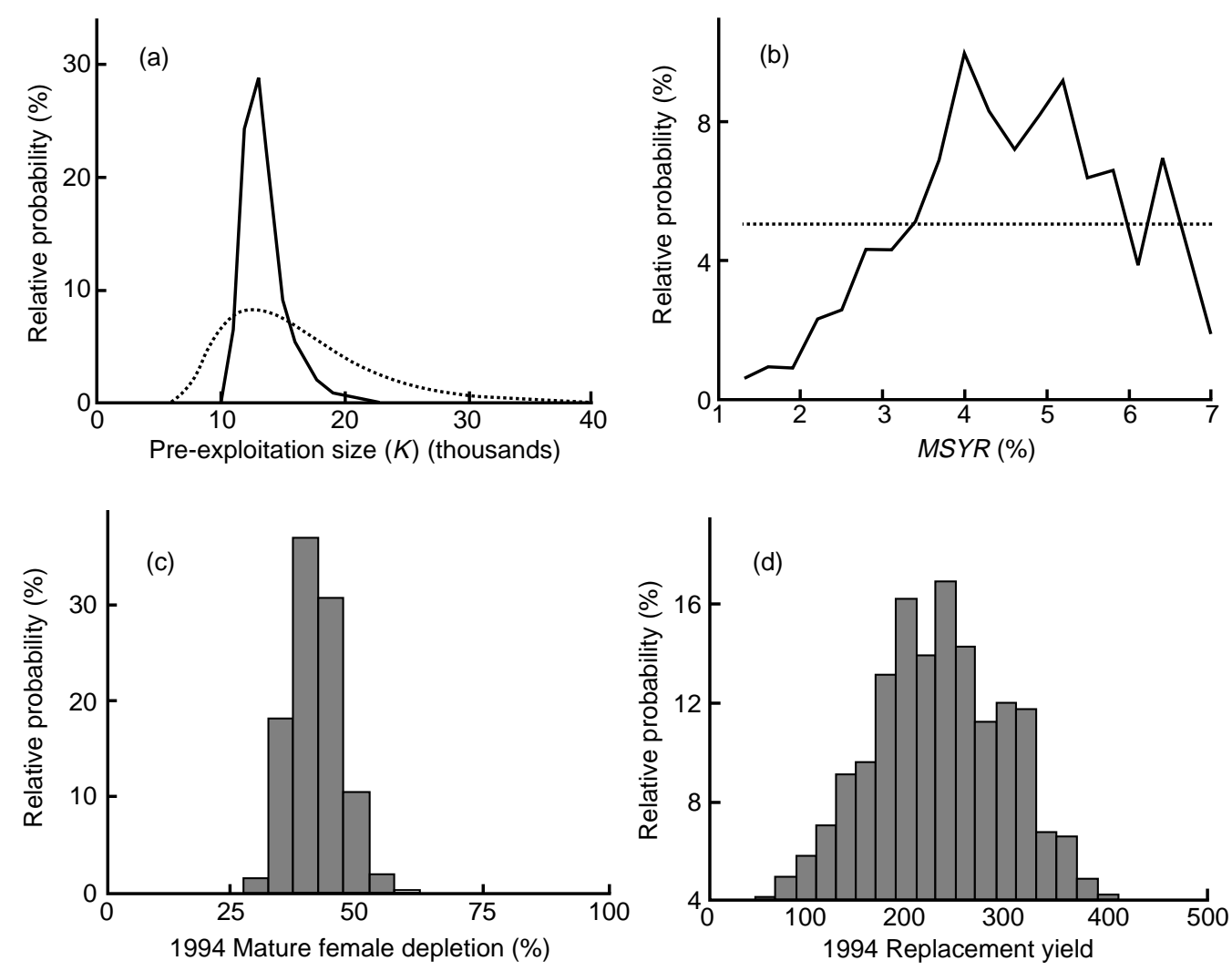

Fig. 3. Posterior distribution (solid lines and bars) for four quantities of relevance to the management of the Bering-Chukchi-Beaufort Seas stock of bowhead whales. The dotted lines in the two upper panels represent the assumed prior distributions. $M S Y R$ is the ratio of $M S Y$ to the population size at which $M S Y$ is achieved.

population, MSYR, MSYL and $K$. Figure 3 shows the prior and posterior distributions for two of these quantities and posterior distributions for the 1994 mature female depletion and the 1994 replacement yield. The results show that prior for $K$ is updated markedly while the difference between prior for $M S Y R$ and its posterior is relatively small. The posterior distribution for the mature female depletion is relatively tight while that for the 1994 replacement yield is fairly imprecise. These results indicate that, as expected, even if the data are informative about some quantities, the prior will not necessarily be updated markedly for all the quantities of interest. The results of the bowhead assessment have been used as the basis for an examination of the performances of alternative decision rules for the bowhead population by Punt and Polacheck (1996).

Figure 4(a) shows the posterior distribution for the current (1994) biomass of hoki while Fig. 4(b) provides the posterior distribution for this biomass expressed as a percentage of the virgin biomass. The results in Fig. 4 (a) and (b) are based on an assessment that places a uniform prior on $B_{0}$ from 0 to 3 million tonnes and restricts the current depletion to lie between 0 and 1 . 

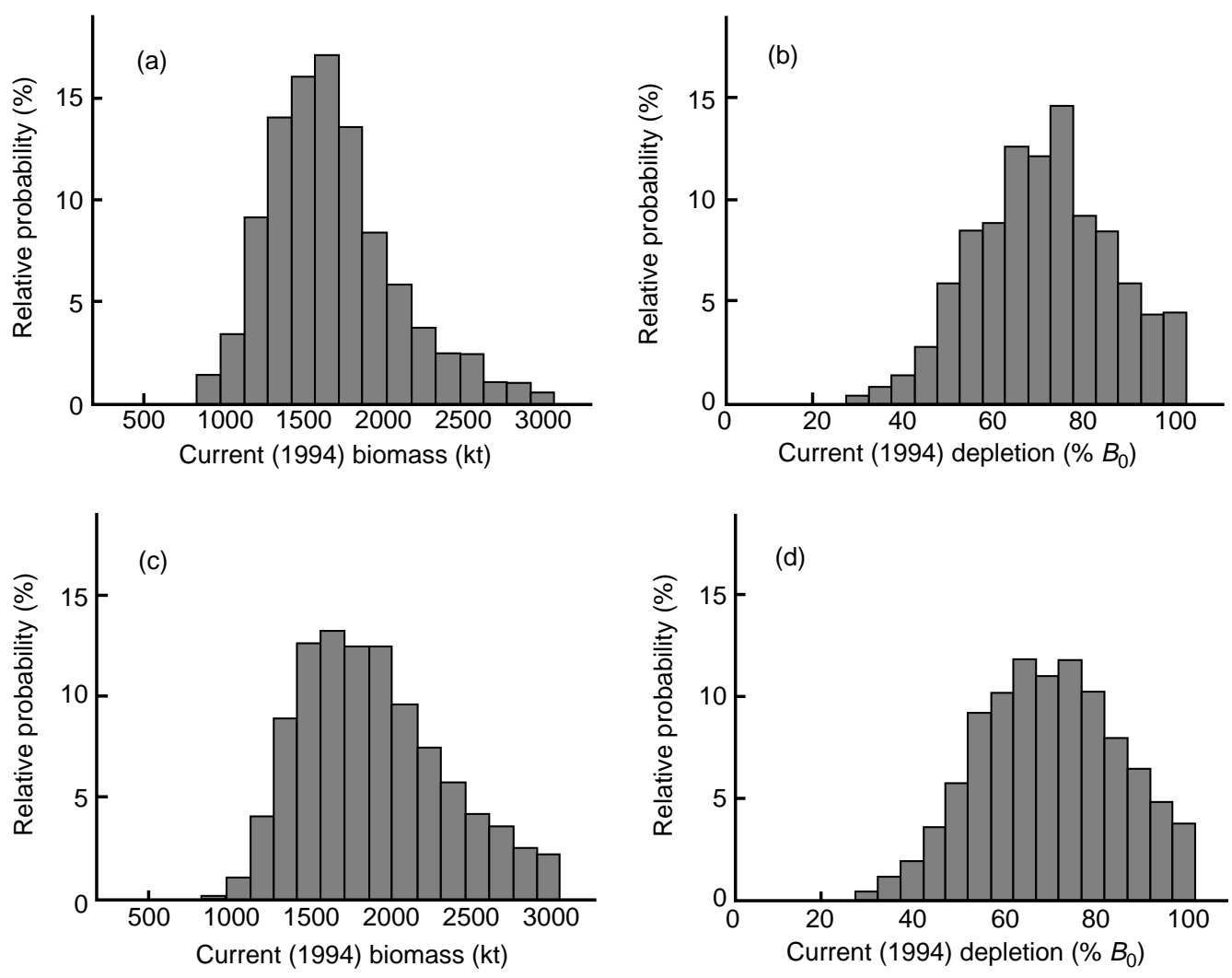

Fig. 4. Posterior distributions for the current biomass and current depletion of the hoki stock from two Bayesian assessments. The results in the upper panels are based on a $\mathrm{U}[0,3000 \mathrm{kt}]$ prior for $B_{0}$ while those in the lower panels are based on a $U[0,1]$ prior for current depletion.

The results in Fig. 4 were used as input to a decision analysis to assess the impact of various alternative future series of catch quotas. The performance indicators chosen to quantify risk are the probability that the mid-season biomass of mature animals drops below $20 \%$ of its pre-exploitation equilibrium level, 'risk (stock)', and the probability that the industry is unable to take at least $80 \%$ of its catch quota, 'risk (catch)' (Annala, 1994). Table 2 lists performance indices for four alternative series of catch

Table 2. Performance indices (see text for definitions) for hoki. The quota is assumed to be fixed for 5 years starting in 1995

\begin{tabular}{lcccc}
\hline Quantity & \multicolumn{4}{c}{ Annual quota $\left(10^{3} \mathrm{t}\right)$} \\
\cline { 2 - 5 } & 200 & 300 & 400 & 500 \\
\hline Risk (stock) & 0.037 & 0.160 & 0.360 & 0.541 \\
Risk (catch) & 0.002 & 0.061 & 0.230 & 0.427 \\
$E(C)$ & 200 & 297 & 383 & 450 \\
$P\left(B_{\text {fin }} . B_{M S Y}\right)$ & 0.866 & 0.726 & 0.564 & 0.406 \\
\hline
\end{tabular}


quotas. Results are shown for the two risk statistics as well as the expected annual catch, $E(C)$, and the probability that the biomass at the end of the 5 year projection period, $B_{\text {fin }}$, exceeds that at which $M S Y$ is achieved, $P\left(B_{f i n} . B_{M S Y}\right)$. Note that the annual catch may differ from the specified catch quota if the stock is driven to low levels so the quota cannot be taken. Table 2 differs from the type of decision table mentioned earlier because the uncertainty inherent in the posterior distribution for the population parameters has been 'integrated out'. The results in Table 2 illustrate the trade-off between risk and reward which decision makers have to evaluate to select a quota which appropriately balances the desire for high yields and the satisfaction of the objective of maintaining a healthy spawning population.

\section{Discussion}

\section{ADVANTAGES AND DISADVANTAGES OF THE BAYESIAN APPROACH}

The main reason for using a Bayesian approach to stock assessment is that it facilitates representing and taking account of the full range of uncertainties related to models and parameter values. In contrast, most decision analyses based on conditional maximum likelihood (or least squares) estimation involve fixing (conditioning on) the values of parameters that may, in actuality, have an important bearing on the final outcome of the analysis and for which there is considerable uncertainty. For example, Francis et al. (1992) contrast assessments that include and ignore the effects of interannual fluctuations in recruitment by examining the posterior distribution for $B_{0}$, and find that ignoring this uncertainty leads to considerable underestimation of the uncertainty about $B_{0}$.

In the past, the effects of uncertainty have been evaluated through sensitivity analysis. In general, this involved changing the value of a single parameter only and rerunning the entire stock assessment. This limitation to a single parameter was due to time constraints and was needed to avoid large amounts of model output. There is clearly a need for sensitivity analysis for any stock assessment. However, current practice cannot guarantee that some (reasonably plausible) combination of parameter values does not give rise to behaviour that would not be expected from the results of sensitivity tests which involve changing the value of a single parameter only. In addition, it is often difficult to summarize the management implications of sensitivity tests that exhibit considerable sensitivity without some form of integration across those tests. In contrast, the Bayesian approach to stock assessment explicitly allows for weighting across alternative states of nature through Bayes Theorem, although this can 'hide' those sets of parameter values that do give rise to poor performance. The use of Bayesian techniques does not eliminate the need for sensitivity tests. It is still necessary to conduct an extensive examination of the sensitivity of the stock assessment and decision analysis results to the choice of the prior distributions, the data set choices, etc. Givens et al. (1994) describe a computationally efficient reweighting approach for assessing sensitivity in Bayesian stock assessments which can be applied in conjunction with the SIR algorithm and would facilitate such an examination.

Current stock assessments, both Bayesian and non-Bayesian, tend to ignore the true range of uncertainty (both model and parameter). In particular, model-structure uncertainty is usually completely ignored (Sainsbury, 1988, is a notable exception) even though the impact of this source of uncertainty can be more important than that of 
uncertainty about the values for the parameters for any one model - this is particularly the case if the future consequences of management actions are to be evaluated.

The process of eliciting prior distributions can be very time consuming and frustrating. Scientists who are experts with the species concerned but who are unaware of Bayesian techniques (and hence do not have a full understanding of what is meant by a prior distribution) can provide 'prior distributions' that are either inconsistent or far too precise (Walters and Ludwig, 1994). However, this process is comparable with that of selecting a 'base case' assessment and a set of sensitivity tests as is common when conducting assessments based on maximum likelihood methods. Although 'expert' opinion is currently the dominant method for determining priors and this is subject to many problems, we believe that prior distributions will increasingly be determined by analysis of information from synthesis studies and hence will depend less on 'expert' opinion. The majority of the problems encountered during the development of the hoki and bowhead whale assessments have resulted from arguments about the choice of prior distributions. In particular, considerable difficulties arose in both cases when attempts were made to select appropriate noninformative prior distributions. In contrast, the development of informative priors tended to be productive, with most participants in the stock assessment groups concerned cooperating even in fairly confrontational assessment situations.

Care needs to be taken to avoid specifying contradictory priors (i.e. specifying two (or more) priors for the same model parameter). This can occur if priors are specified for many quantities which are linked via the population dynamics model. Consider, for example, a situation for the hoki stock in which the following two priors are specified: (a) $B_{0}$ is uniformally distributed from 0 to $3000 \mathrm{kt}$; and (b) the current depletion is uniformally distributed from 0 to 1 . These two priors appear to be both sensible and a reflection of a lack of information. However, they are effectively providing information about the same model quantity, namely the biomass of the population. Figure 4 provides results for two different ways of including these priors in an analysis. The first assessment (Fig. 4 (a) and (b)) is based on a uniform prior for $B_{0}$ from 0 to $3000000 \mathrm{t}$ and restricts the current depletion to lie between 0 and 1 , while the other assessment (Fig. 4 (c) and (d)) corresponds to assuming a U[0, 1] prior for current depletion and restricts the value of $B_{0}$ to the range [0, $3000000 \mathrm{t}$ ]. The prior of $B_{0}$ (Fig. 4 (a) and (b)) leads to more optimistic results because of the implicit prior which a prior on $B_{0}$ places on the current biomass (Fig. 2). Although the results are clearly different, the management implications of likely alternative catch quotas do not differ markedly.

The assessment data for hoki are relatively informative. Had the data been less informative, the results would have differed to an even greater extent, with the prior on $B_{0}$ indicating a resource close to its virgin state and the results for the prior on current depletion indicating that almost nothing is known about the current status of the resource relative to its virgin level. In this example, broadening the range for the $B_{0}$ prior from 3 million to 4 million tonnes would lead to a more optimistic appraisal. However, changing the restriction for the analysis based on the current biomass in this way will not affect the results noticeably. This example is fairly simple in that the link between $B_{0}$ and current depletion is obvious. Care needs to be taken when specifying priors so that they are not contradictory because of some complex pathway through the model. This problem of multiple priors for the same quantity has occurred for the bowhead assessment (e.g. Punt and Butterworth, 1996). 
It is extremely intensive computationally to apply Bayes Theorem to complex models: it often takes days of computer time even on reasonably powerful personal computers. However, to conduct defensible decision analyses for assessments based on maximum likelihood estimation, it is usually necessary to conduct a bootstrap analysis (Restrepo et al., 1992). Such an analysis, although not as intensive computationally as applying Bayes Theorem, can often take several hours on a personal computer. In addition, when applying maximum likelihood methods, there is a need to avoid numerical problems such as locating a local (but not the global) maximum - this can again be very time consuming for the analyst. Raftery et al. (1995) assert that one of the advantages of their Bayesian approach over maximum likelihood methods is the avoidance of convergence problems, which have impeded timely completion of previous assessments.

One of the most frequent criticisms of the stock assessment process is that it is a 'closed shop' and that only scientists fluent in the mathematical methods can contribute (see Introduction in Hilborn, 1992). In contrast, use of Bayesian methods allows scientists with expertise in many other areas to contribute to assessments, primarily through the process of designing prior distributions.

We believe that Bayesian techniques for stock assessment and decision analysis should be the 'methods of choice'. Nonetheless, we are not so naive as to believe that Bayesian methods should be the only methods used. Although Bayesian stock assessments have several advantages over alternative methods, the actual performance of this approach in terms of the bias and variability of estimated quantities is not necessarily better than methods based on maximum likelihood estimation (Cordue and Francis, 1994). As we emphasized earlier with respect to models, there is a need to apply a number of alternative methods. If the results are generally insensitive to model and estimator choice, considerably more weight can be placed on the final outcomes.

The Bayesian approach cannot overcome the issue of which of a variety of (possibility conflicting) data types should be included in an assessment. It provides posterior probabilities for alternative hypotheses, not for the reliability of the data set. In general, as much data as possible must be included in the analysis. The most fruitful approach to handling situations in which there are conflicting sources of information (e.g. increasing catch-rate series and declining trawl indices of abundance) is to conduct analyses for each source separately and present the results to the decision makers (Richards, 1991; Schnute and Hilborn, 1993).

\section{FUTURE NEEDS AND ISSUES}

One of the major benefits of the Bayesian approach is the ability to incorporate prior information - indeed, it demands such information. While other stock assessment approaches use 'prior' information by specifying levels or ranges of individual parameters for use in sensitivity analysis, the Bayesian approach forces the analyst to look at historical data sets to determine what is known about the biological parameters and processes. Within the context of age-structured population dynamics models, we believe that researchers should develop databases to allow the construction of distributions for the rate of natural mortality (Pauly, 1980), the relationship between spawning stock size and subsequent recruitment (McAllister, 1995; Myers et al., 1995), recruitment variability (McAllister, 1995), depensation, etc. Most traditional stock assessment methods do not use any of the quantitative information that could be gathered 
from historical experience with other stocks and, in effect, treat each stock assessment as a new independent problem. Stock assessment scientists need to develop databases that summarize historical experience with other stocks so that it can be used in subsequent assessments.

Finally, a major impediment to the Bayesian approach is computing power. The algorithms we now use (Appendix A) are slow: a typical run using an age-structured model takes between 5 hours and several days on a modern desktop computer. There is an obvious need to stimulate numerical analysis research into more efficient algorithms.

\section{Acknowledgements}

We thank many of our colleagues for useful discussions which led to the developments described in this paper. Specifically, we would like to thank Doug Butterworth, Rick Deriso, Murdoch McAllister, Ellen Pikitch, Keith Sainsbury, Jon Schnute, Carl Walters and Judy Zeh. Tim Hammond, Ulrike Hilborn, David McDonald, Tony Smith and two anonymous reviewers provided extensive comments on earlier drafts of the manuscript.

\section{Appendix A: Methods for numerical integration}

The posterior distribution that results from complicated models can often be characterized only numerically. A variety of numerical methods are available for computing the integrals needed for Bayesian analysis (Smith, 1991, and references therein). We will concentrate on three methods which we have found to be useful even for quite badly behaved and markedly non-normal posterior distributions. Walters and Ludwig (1994) show that it is possible to perform some of the integrals analytically for some prior distributions for the catchability coefficient and its variance (Equation 3). Use of the methods outlined by Walters and Ludwig (1994) is recommended because they reduce the computational demands markedly. The amount of computer time needed to perform the calculations increases geometrically with the number of parameters.

The purpose of the algorithms in this appendix is to sample a set of vectors $\left\{\underline{七}_{i}: i=1,2, \ldots\right\}$ from the posterior distribution, $(\underline{\underline{\iota}}) \propto g(\underline{\mathfrak{t}})=L(D \mid \underline{\underline{ }}) p(\underline{\mathrm{t}})$. This sample can then be used as input to a decision analysis or for inference about the values of quantities of interest to management. Evaluation of $L(D \mid \underline{\underline{ }})$ involves projecting the age-structured population dynamics model forward, using known catches, to predict stock biomasses and then calculating the likelihood for the projection. If the population becomes extinct before the most recent year, it is customary to set the likelihood to zero. This is equivalent to placing an improper prior on the current depletion which is uniform above 0 .

To gauge the information content of the data, it is necessary to compare the prior distribution for a quantity of interest, such as the current biomass, with a numerical representation of its marginal posterior distribution. This distribution is calculated by dividing the range for the quantity of interest $(f(\underline{\underline{t}}))$ into a large number of discrete 'bins' of equal width. The probability assigned to bin $B$ is calculated by dividing the number of vectors in the sample for which the value of $f(\underline{\underline{t}})$ lies in bin $B$ by the total number of vectors. 
GRID SEARCH

This approach involves dividing the range for each parameter into a number of discrete narrow intervals, and then evaluating $g(\underline{\underline{\underline{t}}})$, the product of the likelihood and the prior, at the centre of each grid square. The sample from the posterior is then obtained by sampling values of $Ł$ from those considered with replacement, assigning a probability of

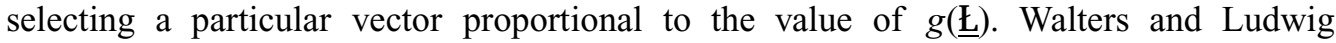
(1994) recommend that the range for each parameter should be divided into at least 40 intervals. Thus, this approach is reasonable for problems with a relatively small number of parameters (up to a maximum of, say, four - at which point the calculation involves over 2.5 million evaluations of the likelihood).

A problem with this approach is that if the data are highly informative or there are high posterior correlations (often the case when age-structure data are used), most of the likelihood evaluations have a negligible contribution to the total probability and it is possible not to sample the area of parameter space with highest support adequately. It is also possible for the same point to be selected a large number of times, leading to a complex multivariate posterior being represented by a small number of points. This can lead to poor estimation of probabilities and hence the quantities needed to conduct the decision analysis. The solution to these problems is to reduce the width of each interval, but this leads to greatly increased computational demands. On the other hand, this approach can deal adequately with multimodal posterior distributions (if the width of each interval is chosen well), which is a problem for the Metropolis method (see below).

This approach is extremely easy to implement. However, the SIR method (see below), which is very similar to the grid search method, is more efficient computationally, especially for problems involving large numbers of parameters.

THE METROPOLIS ALGORITHM (HASTINGS, 1970)

The Metropolis algorithm involves selecting an initial parameter vector $\underline{\underline{~}}_{0}$ and generating

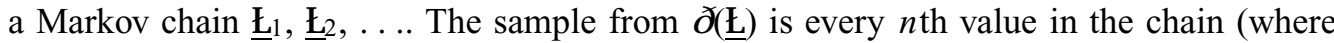
$n$ is selected so that the covariance between $\underline{七}_{i n}$ and $\underline{七}_{(i+1) n}$ is sufficiently small that it can be safely ignored).

The algorithm proceeds by specifying the initial state $\underline{七}_{0}$ (where the vector $\npreceq$ is of

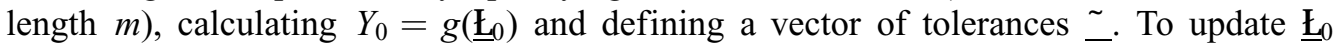
to $\underline{Ł}_{1}$ (or more generally to update $\underline{Ł}_{i}$ to $\underline{Ł}_{i+1}$ ), the following steps are carried out for each element of $\underline{\iota}_{0}\left(\iota_{0, j}\right)$.

1. Generate a 'proposal' $Ł g_{, j}$ from the uniform distribution on the interval $\left[七_{0, j}-{ }_{j}, \iota_{0, j}+{ }_{j}\right]$.

2. Calculate $Y_{0}=g(\underline{\underline{\theta} \theta)}$.

3. Generate a random variable $U$ from the uniform distribution on the interval $[0,1]$. If $Y \theta / Y_{0}$. U the 'proposal' is accepted, $\underline{\iota}_{1}=\succeq 9$ and $Y_{1}=Y q$, end.

4. The 'proposal' $Ł 9$ was not accepted so $\underline{Ł}_{1}=\underline{七}_{0}$ and $Y_{1}=Y_{0}$, end.

Steps 1-4 (referred to as a cycle) are repeated a large number of times. The vector of tolerances $\sim$ is updated dynamically. An algorithm for doing this is to keep a record of the proportion of times the 'proposal' for element $j$ is accepted, and to increase the $j$ th element of ${ }_{-}$by $1 \%$ if this proportion is greater than 0.5 and vice versa. This updating is 
conducted every 5-10 cycles. The results of the first 1000 or so cycles should be ignored, as this is a 'burn-in' period for the algorithm to set itself up. Note that the use of the uniform distribution to generate proposals is only of several alternatives (Hastings, 1970; Gelman et al., 1995). We have found that the use of a multivariate distribution for this purpose (Gelman et al., 1995) can improve the computational efficiency of the algorithm considerably.

There is considerable debate about how many cycles to conduct (or how to assess whether the chain has converged to the posterior distribution). This point is of importance because, if the algorithm is stopped before the chain reaches convergence, the results can be misleading. Although it is feasible to assess this visually by plotting the values of quantities of interest against cycle number and examining the plots for strong autocorrelation (Raftery and Lewis, 1992), Gelman and Rubin (1992) recommend that analyses be conducted for a range of initial parameter vectors instead. If the results from such multiple runs do not agree, it can be concluded that the runs are too short. However, even if there is no disagreement, it cannot be concluded that the runs are sufficiently long. Gelman and Rubin (1992) and Geyer (1992) provide quantitative measures that can be used to assess the extent of convergence.

Another problem with this approach occurs if the posterior is multimodal and the initial state is in one of the modes, but the chain does not reach all of the other modes so that it does not represent an important part of the posterior; an example of such a posterior is given by Gelman and Rubin (1992).

We have found this algorithm to be very computationally efficient for problems with a large number of parameters and a complicated likelihood function. Unlike the SIR algorithm, this algorithm appears to perform adequately for stock assessments that use catch-at-age data.

THE SIR ALGORITHM (RUBIN, 1987; VAN DIJK ET AL., 1987)

The sample-importance-resample (SIR) algorithm is another method that approximates the posterior distribution for high dimensional problems. This algorithm requires a (possibly crude) approximation to the posterior to generate a sample from ( $\underline{\text { }}$ ). This approximation, $\sim(\underline{\underline{\iota}})$, referred to as the importance function, must have non-zero probability wherever ( $\underline{\underline{t}}$ ) has non-zero probability and must be easy to generate from. The simplest choice for $\sim(\underline{\underline{t}})$ is the prior distribution, $p(\underline{\underline{t}})$, although this choice may not be very efficient if the likelihood supports only a small part of $p(\underline{\swarrow})$. The analyses of Bergh and Butterworth (1987), McAllister et al. (1994), Punt et al. (1994) and Raftery et al. (1995) are all based on this choice. The SIR algorithm proceeds as follows.

1. Generate a vector, $\underline{\varliminf}_{i}$, from the distribution $\sim(\underline{\swarrow})$.

2. Calculate $Y_{i}=g\left(\underline{\mathfrak{七}}_{i}\right)$ and the importance sampling weight $w_{i}=Y_{i} /^{\sim}\left(\underline{\mathfrak{七}}_{i}\right)$ where $\sim\left(\underline{\mathfrak{t}}_{i}\right)$

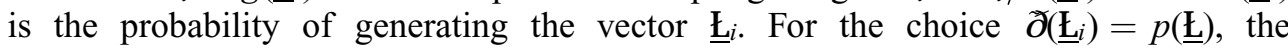
importance sampling weight is simply the likelihood of the data given the vector $\underline{\underline{k}}_{i}$, i.e. $L\left(D \mid \underline{\underline{t}}_{i}\right)$.

3. Repeat steps 1 and 2 a very large number of times.

4. Select a sample from these vectors with replacement, assigning a probability of selecting a particular vector proportional to its importance weight. As a rule of thumb, we have found that steps 1 and 2 need to be applied until no point in the posterior is assigned more than $1 \%$ of the total probability. 
In some cases it is more efficient to iterate the above algorithm, at each iteration replacing the current choice for $\sim(\underline{\underline{ })}$ by the distribution arising from the previous application of the algorithm (Smith et al., 1987).

\section{Appendix B: The age-structured model}

The model assumes that the population is closed with respect to immigration and emigration, relates recruitment to spawner stock size by means of a Beverton-Holt stock-recruit relationship and accounts for recruitment variability explicitly. Natural mortality is assumed to be independent of age and time, and age-specific selectivity is assumed to be time invariant. Models of this form have been used in the assessments of Cape hake (Punt, 1994), albacore tuna (Thunnus alalunga, Scombridae) (Punt et al., 1995a) and orange roughy (Francis, 1992). It is straightforward to generalize the model to incorporate more realistic assumptions, for instance that natural mortality is age and time dependent. Sex structure can also be modelled easily (De la Mare, 1989). However, such extensions are ignored here for ease of presentation.

The age-specific dynamics of the population are governed by the equation:

$$
N_{y+1, a}= \begin{cases}{\left[B_{y+1}^{s} /\left(Æ \mathbb{E}+B_{y+1}^{s}\right)\right] \quad y+1} & a=0 \\ N_{y, a-1} e^{-\left(M+S_{a-1} F_{y}\right)} & 1<a<x-1 \\ N_{y, x-1} e^{-\left(M+S_{x-1} F_{y}\right)}+N_{y, x} e^{-\left(M+S_{x} F_{y}\right)} & a=x\end{cases}
$$

where $N_{y, a}$ is the number of animals of age $a$ at the start of year $y, B_{y}^{s}$ is the spawner stock size at the start of year $y$ :

$$
B_{y}^{s}=\sum_{a=1}^{x} f_{a} N_{y, a}
$$

$f_{a}$ is the number of eggs produced by an animal of age $a$, i.e. the fecundity of an animal of age $a, y$ is the 'recruitment multiplier' for year $y, €$ are the stock-recruit relationship parameters, $M$ is the instantaneous rate of natural mortality, $S_{a}$ is the selectivity of the fishing gear on a fish of age $a, F_{y}$ is the fully selected $\left(S_{a} \rightarrow 1\right)$ fishing mortality during year $y$, and $x$ is the maximum (lumped) age class. Recruitment fluctuation is accounted for by the introduction of $y$ which measures the difference between the realized recruitment and the value expected from the stock-recruit relationship.

Were there no fluctuations in births (i.e. $y=1$ ), the resource would be assumed to be at its pre-exploitation level, with the corresponding age structure, at the start of harvesting (year $y_{1}$ ). Instead, because of historical fluctuations in births, the sizes of the cohorts at the start of year $y_{1}$ are drawn from distributions that allow for this fluctuation, and the initial biomass is thus similarly distributed about the corresponding deterministic pre-exploitation level. The initial numbers at age are given by the equations:

$$
\begin{aligned}
& N_{y_{1}, a}=R_{0} \quad{ }_{a} e^{-a M} \quad 0<a<x-1 \\
& N_{y_{1}, x}=R_{0} \frac{e^{-x M}}{1-e^{-M}} \quad a=x
\end{aligned}
$$

where $R_{0}$ is the number of 0 -year-olds at the deterministic equilibrium that corresponds 
to an absence of harvesting, and $\quad{ }_{a}$ is the recruitment multiplier for age $a$. Note that the equation for the plus-group does not incorporate a recruitment variability term, because this group comprises a large number of age classes which will largely damp out this effect. The value of $R_{0}$ is calculated from the value for the average pre-exploitation equilibrium exploitable biomass at the start of the year, $B_{0}$, using the equation:

$$
R_{0}=B_{0} /\left\{\sum_{a=0}^{x-1} w_{a} S_{a} e^{-a M}+w_{x} S_{x} \frac{e^{-x M}}{1-e^{-M}}\right\}
$$

where $w_{a}$ is the weight of a fish of age $a$ at the start of the year.

The stock-recruit relationship is often re-parameterized in terms of $B_{0}$ and the 'steepness' of the stock-recruit relationship ('steepness' is defined as the fraction of the virgin number of births expected when the spawner stock size is reduced to $20 \%$ of its virgin size (Francis, 1992)):

$$
\begin{aligned}
\mathbb{E} & =\frac{(1-h) \tilde{B}_{0}}{4 h} \\
& =\frac{(5 h-1) \tilde{B}_{0}}{4 h B_{0}} \\
\tilde{B}_{0} & =\sum_{a=1}^{x-1} f_{a} e^{-a M}+f_{m} \frac{e^{-x M}}{1-e^{-M}}
\end{aligned}
$$

The fishing mortality during year $y, F_{y}$, is obtained by solving the equation:

$$
C_{y}=\sum_{a=0}^{x} \frac{w_{a+1 / 2} S_{a} F_{y} N_{y, a}\left(1-e^{-\left(M+S_{a} F_{y}\right)}\right)}{M+S_{a} F_{y}}
$$

where $C_{y}$ is the catch (in weight) during year $y$.

\section{References}

Annala, J.H. (comp.) (1994) Report from the Fishery Assessment Plenary, May 1994: stock assessments and yield estimates. (Unpublished report held in NIWA library, Wellington.). $242 \mathrm{pp}$.

Baird, J.W. (1983) A method to select optimum numbers for aging in a stratified approach. In Doubleday, W.G. and Rivard, D., eds. Sampling Commercial Catches of Marine Fish and Invertebrates. Can. Spec. Publ. Fish. Aquat. Sci. 66, 161-4.

Baker, C.S. (1995) Estimation of rates of annual increase (ROI) of the Bering-Chukchi-Beaufort Seas stock of bowhead whales for the years 1985-93. Rep. Int. Whal. Commn 45, 162-3.

Bergh, M.O. and Butterworth, D.S. (1987) Towards rational harvesting of the South African anchovy considering survey imprecision and recruitment variability. S. Afr. J. Mar. Sci. 5, 937-51.

Bernardo, J.M. (1979) Reference posterior distributions for Bayesian inference. J. R. Statist. Soc. 41B, $113-47$

Best, P.B. (1990) Recovery rates in whale stocks that have been protected from commercial whaling for at least 20 years. Rep. Int. Whal. Commn 40, 129-30.

Best, P.B. (1993) Increase rates in severely depleted stocks of baleen whales. ICES J. Mar. Sci. 50, $169-86$.

Bockstoce, J.R. and Botkin, D.B. (1983) The historical status and reduction of the western Arctic bowhead whale (Balaena mysticetus) population by the pelagic whaling industry. 1848-1914. Rep. Int. Whal. Commn (Spec. Iss. 5), 107-41. 
Box, G.E.P. and Tiao, G.C. (1973) Bayesian Inference in Statistical Analysis. New York: Wiley. $588 \mathrm{pp}$.

Butterworth, D.S. (1995) On reservations concerning the results of the Bayesian synthesis analysis, given the approach used to develop prior distributions for natural mortality rates. Rep. Int. Whal. Commn 45, 163-4.

Butterworth, D.S. and Bergh, M.O. (1993) The development of a management procedure for the South African anchovy resource. In Smith, S.J., Hunt, J.J. and Rivard, D. eds. Risk Evaluation and Biological Reference Points for Fisheries Management. Can. Spec. Publ. Fish. Aquat. Sci. 120, 83-99.

Butterworth, D.S. and Punt, A.E. (1995) On the Bayesian approach suggested for the assessment of the Bering-Chukchi-Beaufort Seas stock of bowhead whales. Rep. Int. Whal. Commn 45, $303-11$.

Butterworth, D.S., De Oliveria, J.A.A. and Cochrane, K.L. (1993) Current initiatives in refining the management procedure for the South African anchovy resource. In Kruse, G., Eggers, D.M., Marasco, R.J., Pautzke, C. and Quinn, T.J. II, eds. Proc. Int. Symp. Management Strategies for Exploited Fish Populations. Fairbanks, AL: Alaska Sea Grant University of Alaska Fairbanks, College Program Report No. 93-02, pp. 439-73.

Butterworth, D.S., Punt, A.E. and Smith, A.D.M. (1996) On plausible hypotheses and their weightings, with implications for selection between variants of the Revised Management Procedure. Rep. Int. Whal. Commn 46, 637-40.

Collie, J.S. and Walters, C.J. (1991) Adaptive management of spatially replicated groundfish populations. Can. J. Fish. Aquat. Sci. 48, 1273-84.

Cordue, P.L. and Francis, R.I.C.C. (1994) Accuracy and choice in risk estimation for fisheries assessment. Can. J. Fish. Aquat. Sci. 51, 817-29.

Cushing, D.H. (1982) Climate and Fisheries. New York: Academic Press. 373 pp.

De la Mare, W.K. (1989) The model used in the Hitter and Fitter program. Rep. Int. Whal. Commn 39, $150-1$.

Delury, D.B. (1947) On the estimation of biological populations. Biometrics 3, 145-67.

Deriso, R.B. (1980) Harvesting strategies and parameter estimation for an age-structured model. Can. J. Fish. Aquat. Sci. 37, 268-82.

Francis, R.I.C.C. (1992) Use of risk analysis to assess fishery management strategies: a case study using orange roughy (Hoplostethus atlanticus) on the Chatham Rise, New Zealand. Can. J. Fish. Aquat. Sci. 49, 922-30.

Francis, R.I.C.C., Robertson, D.A., Clark, M.R. and Coburn, R.P. (1992) Assessment of the ORH 3B orange roughy fishery for the 1992/93 fishing year. New Zealand Fish. Assess. Res. Doc. 92/4, $45 \mathrm{pp}$.

Gavaris, S. (1988) An adaptive framework for the estimation of population size. Can. Atl. Fish. Sci. Adv. Comm. (CAFSAC) Res. Doc. 88/29, 12 pp.

Gavaris, S. and Gavaris, C.A. (1983) Estimation of catch at age and its variance for groundfish stocks in the Newfoundland region. In Doubleday, W.G. and Rivard, D., eds. Sampling Commercial Catches of Marine Fish and Invertebrates. Can. Spec. Publ. Fish. Aquat. Sci. 66, 178-82.

Gelman, A. and Rubin, D.B. (1992) Inference from iterative simulation using multiplicative sequences. Statistical Sci. 7, 457-72.

Gelman, A., Carlin, J.B., Stern, H.S. and Rubin, D.B. (1995) Bayesian Data Analysis. London: Chapman and Hall. 526 pp.

Geyer, C.J. (1992) Practical Markov chain Monte Carlo. Statistical Sci. 7, 473-83.

Givens, G.H., Raftery, A.E. and Zeh, J.E. (1993) Benefits of a Bayesian approach for synthesizing multiple sources of evidence and uncertainty linked by a deterministic model. Rep. Int. Whal. Commn 43, 495-500.

Givens, G.H., Raftery, A.E. and Zeh, J.E. (1994) A reweighting approach for sensitivity analysis within the Bayesian synthesis framework for population assessment modeling. Rep. Int. Whal. 
Commn 44, 377-84.

Hastings, W.K. (1970) Monte Carlo sampling methods using Markov chains and their applications. Biometrika 57, 97-109.

Hilborn, R. (1992) Current and future trends in fisheries stock assessment and management. In Payne A.I.L., Brink, K.H., Mann, K.H. and Hilborn, R., eds. Benguela Trophic Functioning. S. Afr. J. Mar. Sci. 12, 975-88.

Hilborn, R. and Luedke, W. (1987) Rationalizing the irrational: a case study in user group participation in Pacific salmon management. Can. J. Fish. Aquat. Sci. 44, 1796-1805.

Hilborn, R. and Walters, C.J. (1992) Quantitative Fisheries Stock Assessment: Choice, Dynamics and Uncertainty. London: Chapman and Hall. 570 pp.

Hilborn, R., Pikitch, E.K. and McAllister, M.K. (1994) A Bayesian estimation and decision analysis for an age-structured model using biomass survey data. Fish. Res. 19, 17-30.

Hoenig, J.M., Warren, W.G. and Stocker, M. (1994) Bayesian and related approaches to fitting surplus production models. Can. J. Fish. Aquat. Sci. 51, 1823-31.

IWC (International Whaling Commission) (1989) The Comprehensive Assessment of Whale Stocks: The Early Years. Rep. Int. Whal. Commn (Spec. Iss. 11), 210 pp.

IWC (International Whaling Commission) (1990) Report of the Scientific Committee, Annex F. Report of the Sub-Committee on Biological Parameters and MSY Rates. Rep. Int. Whal. Commn 40, $119-30$

IWC (International Whaling Commission) (1993) Report of the Scientific Committee, Annex I. Report of the Working Group on Implementation Trials. Rep. Int. Whal. Commn 43, 153-96.

IWC (International Whaling Commission) (1995) Report of the Scientific Committee, Annex F. Report of the Sub-Committee on Aboriginal Subsistence Whaling. Rep. Int. Whal. Commn 45, 142-64.

Jeffreys, H. (1961) Theory of Probability, 3rd edn. Oxford: Clarendon Press. 447 pp.

Kinas, P.G. (1993) Bayesian statistics for fishery stock assessment and management. PhD thesis, Univ. British Columbia, Vancouver.

Liermann, M. and Hilborn, R. (1997) Depensation in fish stocks: a hierarchic Bayesian meta-analysis. Can. J. Fish. Aquat. Sci. (in press)

Lindley, D.V. (1983) Theory and practice of Bayesian statistics. The Statistician 32, 1-11.

McAllister, M.K. (1995) Using decision analysis to choose a design for surveying fisheries resources. $\mathrm{PhD}$ thesis, Univ. Washington, Seattle. 293 pp.

McAllister, M.K., Pikitch, E.K., Punt, A.E. and Hilborn, R. (1994) A Bayesian approach to stock assessment and harvest decisions using the sampling/importance resampling algorithm. Can. J. Fish. Aquat. Sci. 51, 2673-87.

Methot, R.D. (1989) Synthetic estimates of historical abundance and mortality for northern anchovy. Am. Fish. Soc. Symp. 6, 66-82.

Myers, R.A., Bridson, J. and Barrowman, N.J. (1995) Summary of worldwide spawner and recruitment data. Can. Tech. Rep. Fish. Aquat. Sci. No. 2024, 327 pp.

Pauly, D. (1980) On the interrelationships between natural mortality, growth parameters, and mean environmental temperature in 175 fish stocks. J. Cons. Int. Explor Mer 39, 175-92.

Pelletier, D. and Laurec, A. (1992) Management under uncertainty: defining strategies for reducing overexploitation. ICES J. Mar. Sci. 49, 389-401.

Powers, J.E. and Restrepo, V.R. (1992) Additional options for age-sequenced analysis. Col. Vol. Sci. Pap. ICCAT 39, 540-53.

Punt, A.E. (1992) Selecting management methodologies for marine resources, with an illustration for southern African hake. In Payne, A.I.L., Brink, K.H., Mann, K.H. and Hilborn, R., eds. Benguela Trophic Functioning. S. Afr. J. Mar. Sci. 12, 943-58.

Punt, A.E. (1993) The comparative performance of production-model and ad hoc tuned VPA based feedback-control management procedures for the stock of Cape hake off the west coast of South Africa. In Smith, S.J., Hunt, J.J. and Rivard, D., eds. Risk Evaluation and Biological Reference Points for Fisheries Management. Can. Spec. Publ. Fish. Aquat. Sci. 120, $283-99$. 
Punt, A.E. (1994) Assessments of the stocks of Cape hake Merluccius spp. off South Africa. S. Afr. J. Mar. Sci. 14, 159-86.

Punt, A.E. and Butterworth, D.S. (1991) On an approach for comparing the implications of alternative fish stock assessments, with application to the stock of Cape hake off northern Namibia. $S . A f r . J$. Mar. Sci. 10, 219-40.

Punt, A.E. and Butterworth, D.S. (1996) Further remarks on the Bayesian approach for assessing the Bering-Chukchi-Beaufort Seas stock of bowhead whales. Rep. Int. Whal. Commn 46, 481-91.

Punt, A.E. and Polacheck, T. (1996) Some thoughts related to the selection of a catch limit algorithm for a revised Aboriginal Subsistence Whaling Scheme. Rep. Int. Whal. Commn 46, 463-73.

Punt, A.E., Pikitch, E.K., McAllister, M.K. and Hilborn, R. (1993) Stock assessment and decision analysis for the western stock of hoki (Macruronus novaezelandie) for 1993. New Zealand Fish. Assess. Res. Doc. 93/13, 39 pp.

Punt, A.E., McAllister, M.K., Pikitch, E.K. and Hilborn, R. (1994) Stock assessment and decision analysis for hoki (Macruronus novaezelandie) for 1994. New Zealand Fish. Assess. Res. Doc. 94/13, $47 \mathrm{pp}$.

Punt, A.E., Butterworth, D.S. and Penney, A.J. (1995a) Stock assessment and risk analysis for the south Atlantic population of albacore Thunnus alalunga using an age-structured production model. S. Afr. J. Mar. Sci. 16, 361-71.

Punt, A.E., Butterworth, D.S. and Martin, J. (1995b) The effects of errors in the placement of the boundary between the West and South Coast hake Merluccius spp. stocks on the performance of the current hake management procedure. S. Afr. J. Mar. Sci. 15, 83-98.

Raftery, A.E. and Lewis, S.M. (1992) Comment: on long runs with diagnostics: implementation strategies for Markov chain Monte Carlo. Statistical Sci. 7, 493-7.

Raftery, A.E., Givens, G.H. and Zeh, J.H. (1995) Inference from a deterministic population dynamics model for bowhead whales. J. Am. Statist. Assoc. 90, 402-16.

Restrepo, V.R., Hoenig, J.M., Powers, J.E., Baird, J.W. and Turner, S.C. (1992) A simple simulation approach to risk and cost analysis, with applications to swordfish and cod fisheries. Fishery Bull., Wash. 90, 736-48.

Richards, L.J. (1991) Use of contradictory data sources in stock assessments. Fish. Res. 11, 225-38.

Richards, L.J., Schnute, J.T., Kronlund, A.R. and Beamish, R.J. (1992) Statistical models for the analysis of ageing error. Can. J. Fish. Aquat. Sci. 49, 1801-15.

Ricker, W.E. (1975) Computation and interpretation of biological statistics of fish populations. Bull. Fish. Res. Board Can. No. 191. 382 pp.

Rubin, D.B. (1987) Comment: the calculation of posterior distributions by data augmentation. J. Am. Statist. Assoc. 82, 543-6.

Sainsbury, K.J. (1988) The ecological basis of multispecies fisheries, and management of a demersal fishery in tropical Australia. In Gulland, J.A., ed. Fish Population Dynamics, 2nd edn. New York: Wiley, pp. 349-82.

Schaefer, M.B. (1954) Some aspects of the dynamics of populations important to the management of commercial marine fisheries. Bull. Inter-Am. Trop. Tuna Commn 1, 25-56.

Schaefer, M.B. (1957) A study of the dynamics of fishery for yellowfin tuna in the Eastern tropical Pacific Ocean. Bull. Inter-Am. Trop. Tuna Commn 2, 247-85.

Schnute, J.[T.] (1985) A general theory for analysis of catch and effort data. Can. J. Fish. Aquat. Sci. 42, 414-29.

Schnute, J.T. and Hilborn, R. (1993) Analysis of contradictory data sources in fish stock assessments. Can. J. Fish. Aquat. Sci. 50, 1916-23.

Schnute, J.T. and Richards, L.J. (1995) The influence of error on population estimates from catch-atage models. Can. J. Fish. Aquat. Sci. 52, 2063-77.

Smith, A.D.M. (1993) Risks of over- and under-fishing new resources. In Smith, S.J., Hunt, J.J. and Rivard, D., eds. Risk Evaluation and Biological Reference Points for Fisheries Management. Can. Spec. Publ. Fish. Aquat. Sci. 120, 261-7. 
Smith, A.F.M. (1991) Bayesian computational methods. Trans. R. Soc. Lond. 337A, 369-86.

Smith, A.F.M., Skene, A.M., Shaw, J.E.H. and Naylor, J.C. (1987) Progress with numerical and graphical methods for practical Bayesian statistics. The Statistician 36, 75-82.

Smith, S.J., Hunt, J.J. and Rivard, D. (eds) (1993) Risk Evaluation and Biological Reference Points for Fisheries Management. Can. Spec. Publ. Fish. Aquat. Sci. 120, 442 pp.

Sullivan, K.J., Cordue, P.L. and Ballara, S.L. (1995) A review of the 1992-93 hoki fishery and assessment of hoki stocks for 1994. New Zealand Fish. Assess. Res. Doc. 95/5, 43 pp.

Thompson, G.G. (1992) A Bayesian approach to management advice when stock-recruitment parameters are uncertain. Fish. Bull., Wash. 90, 561-73.

Van Dijk, H.K., Hop, J.P. and Louter, A.S. (1987) An algorithm for the computation of posterior moments and densities using simple importance sampling. The Statistician 36, 83-90.

Walters, C.J. and Hilborn, R. (1976) Adaptive control of fishing systems. J. Fish. Res. Bd Can. 33, $145-59$

Walters, C.[J.] and Ludwig, D. (1994) Calculation of Bayes posterior probability distributions for key population parameters. Can. J. Fish. Aquat. Sci. 51, 713-22.

Walters, C.J. and Punt, A.E. (1994) Placing odds on sustainable catch using virtual population analysis and survey data. Can. J. Fish. Aquat. Sci. 51, 946-58.

Wolpert, R.L. (1995) Comment: inference from a deterministic population dynamics model for bowhead whales. J. Am. Statist. Assoc. 90, 426-7.

Accepted 3 December 1996 